\title{
Signs of Insufficiency Fractures Overlooked in a Patient Receiving Chronic Bisphosphonate Therapy
}

\author{
Wassi Shaikh, MS III, Dan Morris, DO, and Sean Morris, MS IV
}

Chronic bisphosphonate therapy is associated with atypical fractures of the subtrochanteric and proximal femoral diaphyseal regions. Various radiologic imaging signs can identify bisphosphonate-associated stress fractures before they progress to complete fractures. Identifying patients who are at risk and modifying treatment can prevent these fractures from occurring. We present a case study of a patient taking bisphosphonate with 2 years of chronic pain, and characteristic clinical signs of bisphosphonateassociated incomplete stress fractures that went untreated until the patient suffered from a right subtrochanteric complete fracture. Our goal is to make physicians aware of the signs of insufficiency fractures associated with chronic bisphosphonate therapy such that appropriate clinical decisions are made to optimize the quality of patient care. (J Am Board Fam Med 2016;29:404-407.)

Keywords: Bisphosphonates, Case Reports, Orthopedics, Radiology, Subtrochanteric Fractures

Bisphosphonates have been proven to decrease the risk of both vertebral and nonvertebral fractures in postmenopausal women with osteoporosis. ${ }^{1-3}$ However, recent studies have shown that consistent bisphosphonate therapy for more than 3 to 5 years may increase the risk of atypical femoral fractures, in particular subtrochanteric and proximal femoral diaphysis fractures. ${ }^{4-7}$ The subtrochanteric fractures tend to occur bilaterally, are either transverse or oblique, and have minimal comminution. ${ }^{4,5,8,9}$ Osteonecrosis of the jaw has also been reported as a result of prolonged bisphosphonate therapy. ${ }^{10}$

We present a case study of a patient who had alendronate-associated bilateral subtrochanteric femoral stress fractures that went untreated until the patient suffered a complete fracture in the subtrochanteric region of the right femur. A retrospective review of previous emergency department (ED) visits and imaging demonstrate the character-

This article was externally peer reviewed.

Submitted 31 July 2015; revised 9 January 2016; accepted 20 January 2016.

From Wayne State University School of Medicine (WS), Detroit, MI; Department of Orthopedic Surgery (DM), St John Providence Health System, Southfield, MI; School of Medicine (SM), Mercer University, Macon, GA.

Funding: none.

Conflict of interest: none declared.

Corresponding author: Wassi Shaikh, MS III, Wayne State University School of Medicine, 540 East Canfield Street, Detroit, MI 48201 (E-mail: wshaikh@med.wayne.edu). istic presentation of femoral stress fractures associated with bisphosphonate use.

\section{Case Report}

A female in her mid-60s with a medical history of osteopenia presented to the ED complaining of insidious onset of pain in the left thigh. She described the pain as "dull and achy." She was 5' tall and had a body mass index of $25 \mathrm{~kg} / \mathrm{m}^{2}$. Her medical history was also notable for hypertension and hypercholesterolemia. Her current medications included simvastatin $10 \mathrm{mg}$ daily, multivitamins, and alendronate $70 \mathrm{mg}$ once weekly. Of particular importance was her 6-year history of alendronate use for osteoporosis. She was very compliant with her medication protocol. Her anteroposterior (AP) lumbar spine $\mathrm{T}$-score was -1.6 , and her femoral neck $\mathrm{T}$-score was -1.7 . Her surgical history involved a unilateral ligament reconstruction for scapholunate instability and an anterior cervical discectomy and fusion. She denied use of tobacco, alcohol, or recreational drugs. She was otherwise healthy and was relatively active. She did not use a walker or a wheelchair. Her workup encompassed a complete blood count, a basic metabolic panel, and plain-film images including an AP view of the pelvis and 2 views of the left hip (Figure 1). Laboratory testing showed no abnormalities. The radiology report was read as "mild degenerative changes 
Figure 1. Anteroposterior plain-film image of the pelvis, read as negative study (December 5,2012$)$. A closer look reveals lateral cortical thickening of each femur in the proximal diaphysis.

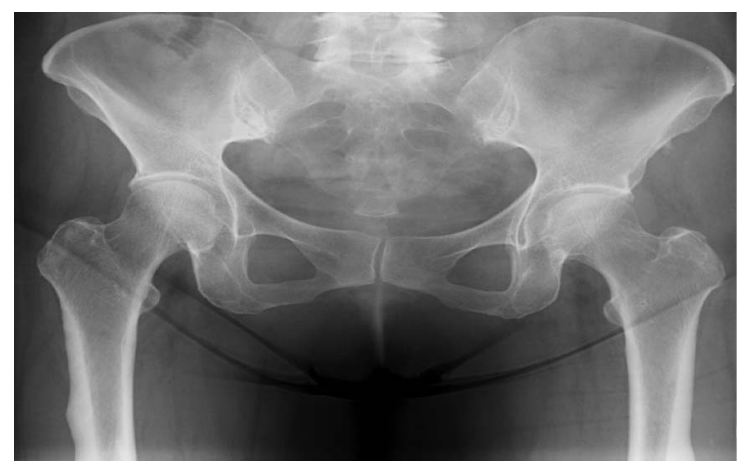

about the lumbar spine and sacroiliac joints. No abnormalities seen about either hip. A recent injury is not identified." The patient was treated symptomatically with pain management and discharged to home.

Approximately 2 years later, the patient returned to the ED with a related complaint of bilateral thigh pain. An AP image of the pelvis, with 2 views of each hip, was read: "the bony pelvis is intact with symmetric sacroiliac joints[.] Femoral head contour is normal. Hip joints are normal, without fracture or dislocation. There is no evidence of soft tissue calcification. Unremarkable study of the pelvis and hips." The patient was again treated for her symptoms and discharged to home.

Six months later, she returned to the ED a third time with a chief complaint of pain in the right thigh after a fall from standing height. She stated that while placing her full weight over her right leg while descending a flight of stairs, she felt a snap and experienced immediate pain that led to her falling onto her right side. She denied loss of consciousness or acute pain at any other site. She indicated that she had been experiencing pain in the right thigh for approximately 6 months and pain in the left thigh for $>2$ years. An AP radiograph of the pelvis (Figure 2) and 2 views of the right hip were read as a "transverse fracture proximal metaphysis of right femur with medial angulation of the distal component." The patient was taken to the operative suite, where a closed reduction was attempted but unsuccessful, followed by successful open fracture reduction and fixation. The patient was advised to not bear weight on her right leg.
Postoperatively, the patient complained of aching pain $(6 / 10)$ on the left side. Radiographs of the left femur were ordered (Figure 3) and read as "focal cortical thickening along the lateral margin of the left femoral diaphysis approximately $13 \mathrm{~cm}$ below the intertrochanteric region of femur. There is a linear lucency in the area of cortical thickening consistent with insufficiency fracture." A bone scan (Figure 4) showed "subtle focal radiotracer activity along the proximal femoral diaphysis of both femurs corresponding to areas of radiographic abnormality. The relatively low-level of radiotracer activity may relate to generalized osseous demineralization and bisphosphonate therapy." Because of the possibility of a fracture in the patient's left leg, the patient was instructed to partially bear weight on her left leg and bear no weight on the right leg, and to use a walker. She was discharged to a rehabilitation facility. Two weeks after surgery the pain in her right leg had improved (2/10), but the pain in the left leg continued as a dull, aching pain $(6 / 10)$.

Six weeks after she was admitted for the right femur fracture, the patient underwent prophylactic fixation of the left femur without complication. Of note, the surgical time was 40 minutes shorter and estimated blood loss was $90 \mathrm{~mL}$ less during the prophylactic fixation of the left femur compared with the earlier open reduction and internal fixation of the right femur. The patient was discharged from the hospital on postoperative day 2 with instructions for weight bearing as tolerated on the left leg and to continue partial weight bearing on

Figure 2. Anteroposterior plain-film image of the pelvis (July 26, 2014) displaying a transverse fracture of the right proximal femoral diaphysis in the area of lateral cortical thickening. The left femur shows lateral cortical thickening in the proximal diaphysis.

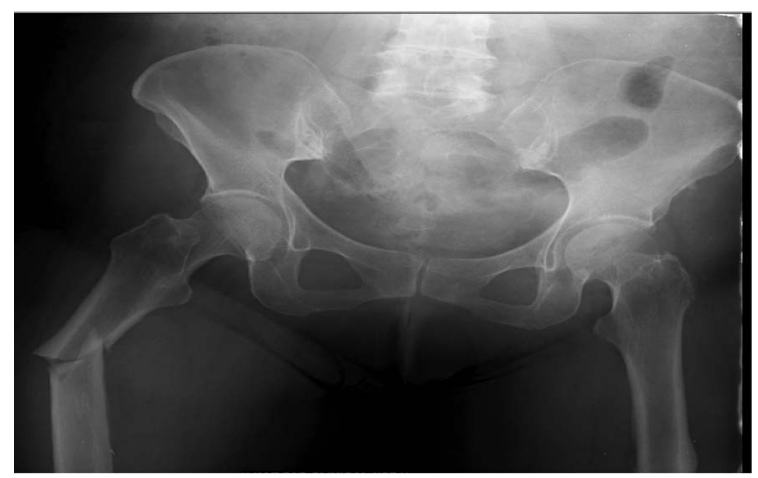


Figure 3. Anteroposterior plain-film image of the left femur (July 28, 2014) shows an area of proximal femoral lateral cortical thickening.

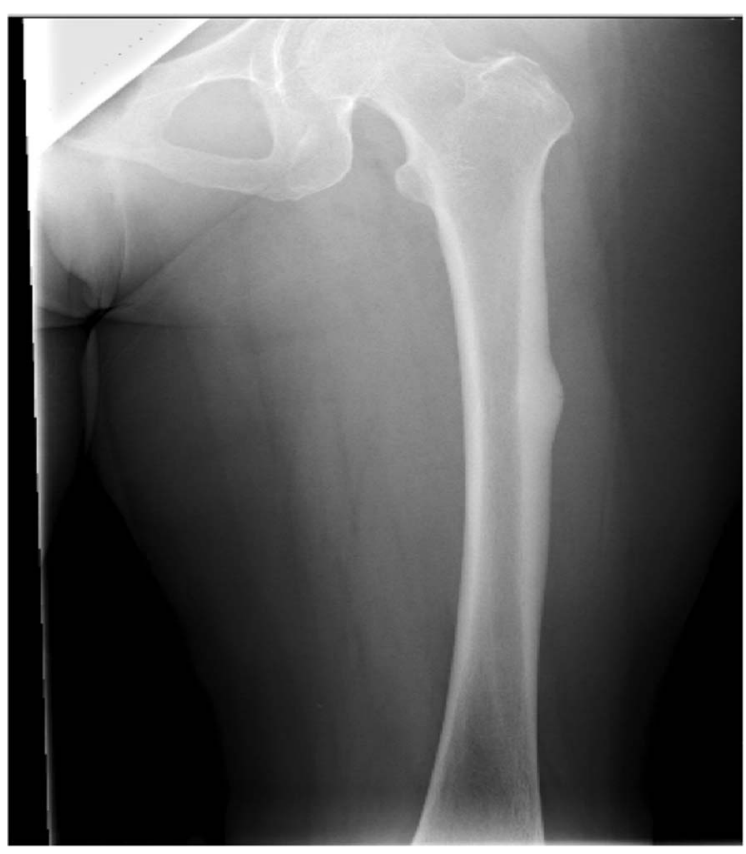

the right leg. With regard to pain and function, the patient exhibited better strength and function with less pain in the prophylactically treated limb at each follow-up visit.

For our literature review, we searched PubMed for the terms bisphosphonate, radiograph, and subtro- chanteric fracture. We selected those references that supported the current evaluation and treatment.

\section{Discussion}

Our patient presented with a transverse subtrochanteric fracture with minimal comminution and lateral cortical thickening, a pattern typical of bisphosphonate-associated fractures. ${ }^{8}$ She had prodromal thigh and groin pain for $>2$ years and a history of nearly 6 years of alendronate therapy for osteoporosis. In retrospect, signs of bisphosphonate-related insufficiency fractures of both femurs could be seen in the films performed as early the patients' first visit to the ED (Figure 1).

For patients with osteopenia or osteoporosis receiving bisphosphonate therapy who have persistent aching thigh and/or groin pain, imaging of the pelvis and femur should be performed, and special care should be given to examining the femur for any signs of insufficiency. The varus angle of the proximal femoral shaft can also be a valuable tool in identifying patients at high risk for atypical femoral fracture. Patients with primary osteoporosis taking chronic bisphosphonate therapy and who have a more varus proximal angle to the femoral shaft are more likely to suffer from an atypical femoral fracture than patients with primary osteoporosis with a less acute angle at the proximal femoral joint. ${ }^{11}$ Special care should be taken to look for signs of

Figure 4. A bone scan (July 29, 2014) showing subtle radiotracer activity along the proximal femoral diaphysis of both femurs.

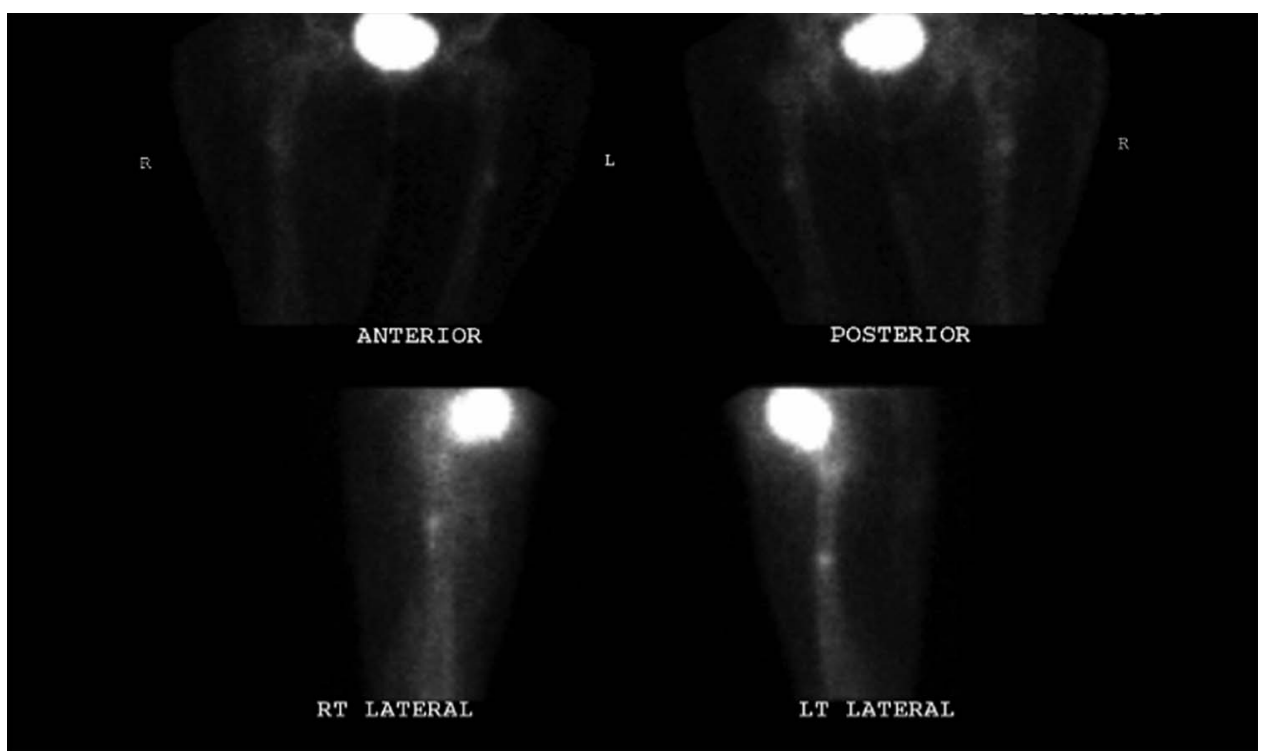


femoral incomplete micro stress fractures, that is, focal or diffuse lateral cortical thickening of the subtrochanter or femoral diaphysis. Once an incomplete fracture progresses, a radiolucent line will be seen. ${ }^{5,6,9}$

There are multiple modalities of treatment for a patient at risk for bisphosphonate-associated fractures. A recent retrospective study suggested that if a symptomatic patient taking long-term bisphosphonate therapy presents with lateral cortical thickening and no radiolucent line-in other words, incomplete stress fractures-then conservative therapy with teriparatide and discontinuation of bisphosphonate treatment may resolve the fracture. ${ }^{12}$ When a radiolucent line, characteristic of a high-risk incomplete atypical fracture, is visible, surgical prophylaxis is recommended to prevent propagation to a complete fracture. ${ }^{5,6,9,12}$ Once the fracture is complete, closed reduction should be attempted first and, if unsuccessful, open reduction and fixation should be performed.

It is important to note that, because of the limited research on bisphosphonate-associated stress fractures, almost all retrospective studies cited involve alendronate. Furthermore, there are only a few patients in each study because of the rarity of atypical femoral fractures. The new Health Outcomes and Reduced Incidence with Zolendonic Acid Once Yearly (HORIZON) Extension trial, conducted by Black et al, ${ }^{7}$ supports similar adverse profiles for bisphosphonate with consistent zoledronic acid treatment for 3 years, which may suggest that physicians consider a cautionary approach to all long-term bisphosphonate therapy until proven otherwise.

\section{Conclusion}

When a patient with a prolonged use of bisphosphonate presents with thigh pain, physicians should include bisphosphonate-associated insufficiency fracture in the differential diagnosis, and adequate diagnostic methods should be used, such as ordering radiologic images of the femur and searching for the subtle lateral cortical thickening of the femoral subtrochanter and/or diaphysis. With femoral insufficiency diagnosed sooner, physicians can intervene earlier with management such as discontinuing/altering bisphosphonates, changing weight bearing, and early prophylactic surgical intervention.

\section{References}

1. Black DM, Schwartz AV, Ensrud KE, et al. Effects of continuing or stopping alendronate after 5 years of treatment: the Fracture Intervention Trial Longterm Extension (FLEX): a randomized trial. JAMA 2006;296:2927-38.

2. Black DM, Cummings SR, Karpf DB, et al. Randomised trial of effect of alendronate on risk of fracture in women with existing vertebral fractures. Fracture Intervention Trial Research Group. Lancet 1996;348:1535-41.

3. Bone HG, Hosking D, Devogelaer JP, et al. Ten years' experience with alendronate for osteoporosis in postmenopausal women. New Engl J Med 2004; 350:1189-99.

4. Goh SK, Yang KY, Koh JS, et al. Subtrochanteric insufficiency fractures in patients on alendronate therapy: a caution. J Bone Joint Surg Br 2007;89: $349-53$.

5. Kwek EB, Goh SK, Koh JS, Png MA, Howe TS. An emerging pattern of subtrochanteric stress fractures: a long-term complication of alendronate therapy? Injury 2008;39:224-31.

6. Lenart BA, Neviaser AS, Lyman S, et al. Association of low-energy femoral fractures with prolonged bisphosphonate use: a case control study. Osteoporos Int 2009;20:1353-62.

7. Black DM, Reid IR, Boonen S, et al. The effect of 3 versus 6 years of zoledronic acid treatment of osteoporosis: a randomized extension to the HORIZONPivotal Fracture Trial (PFT). J Bone Miner Res 2012;27:243-54.

8. Das De S, Setiobudi T, Shen L, Das De S. A rational approach to management of alendronate-related subtrochanteric fractures. J Bone Joint Surg Br 2010; 92:679-86.

9. Neviaser AS, Lane JM, Lenart BA, Edobor-Osula F, Lorich DG. Low-energy femoral shaft fractures associated with alendronate use. J Orthop Trauma 2008;22:346-50.

10. Otto S, Abu-Id MH, Fedele S, et al. Osteoporosis and bisphosphonates-related osteonecrosis of the jaw: not just a sporadic coincidence-a multi-centre study. J Carniomaxillofac Surg 2011;39:272-7.

11. Hagen JE, Miller AN, Ott SM, et al. Association of atypical femoral fractures with bisphosphonate use by patients with varus hip geometry. J Bone Joint Surg Am 2014;96:1905-9.

12. Saleh A, Hegde VV, Potty AG, Schneider R, Cornell $\mathrm{CN}$, Lane JM. Management strategy for symptomatic bisphosphonate-associated incomplete atypical femoral fractures. HSS J 2012;8:103-10. 